TAIWANESE JOURNAL OF MATHEMATICS

Vol. 8, No. 1, pp. 85-102, March 2004

This paper is available online at http://www.math.nthu.edu.tw/tjm/

\title{
EXISTENCE OF SOLUTIONS OF THE $g$-NAVIER-STOKES EQUATIONS
}

\author{
Hyeong-Ohk Bae and Jaiok Roh
}

\begin{abstract}
The $g$-Navier-Stokes equations in spatial dimension 2 are the following equations introuduced in [3]

$$
\frac{\partial \mathbf{u}}{\partial t}-\nu \Delta \mathbf{u}+(\mathbf{u} \cdot \nabla) \mathbf{u}+\nabla p=\mathbf{f}
$$

with the continuity equation

$$
\frac{1}{g} \nabla \cdot(g \mathbf{u})=0
$$

Here, we show the existence and uniqueness of solutions of $g$-Navier-Stokes equations on $\mathbf{R}^{n}$ for $n=2,3$.
\end{abstract}

\section{INTRODUCTION}

The goveming equations for the fluid are the well-known incompressible NavierStokes equations of the form

$$
\frac{\partial \mathbf{u}}{\partial t}-\nu \Delta \mathbf{u}+(\mathbf{u} \cdot \nabla) \mathbf{u}+\nabla p=\mathbf{f}
$$

$$
(\nabla \cdot \mathbf{u})=0
$$

with some initial and boundary conditions. Here, $\nu$ and $f$ are given and the velocity $\mathbf{u}$ and the pressure $p$ are the unknowns. The first equations are called the momentum equations and the second one continuity equation. For the analysis on the NavierStokes equations, refer to [1], [2], [4] and [5].

Consider the Navier-Stokes equations (1.1) and (1.2) on the spatial domain $g:=2 \times[0, g]$, where ${ }_{2}$ is a bounded region in the plane and $g=g\left(x_{1}, x_{2}\right)$ is

Received April 15, 2003.

Communicated by S. B. Hsu.

2000 Mathematics Subject Classification: 35Q10, 76D05.

Key words and phrases: $g$-Navier-Stokes equations, Weak solutions, strong solution, uniqueness.

The first author is supported by the Ajou University Research Fund. 
a smooth function defined on 2 with $0<m \cdot g\left(x_{1}, x_{2}\right) \cdot M$, for $\left(x_{1}, x_{2}\right) \in 2$. The 2D $g$-Navier-Stokes equations have been drived in [3] from the 3D NavierStokes equations on $g$ :

$$
\begin{aligned}
& \frac{\partial \mathbf{u}}{\partial t}-\nu \Delta \mathbf{u}+(\mathbf{u} \cdot \nabla) \mathbf{u}+\nabla p=\mathbf{f} \\
& \frac{1}{g}(\nabla \cdot(g \mathbf{u}))=\frac{\nabla g}{g} \cdot \mathbf{u}+\nabla \cdot \mathbf{u}=0
\end{aligned}
$$

in 2 . Equation (1.3) can be written as

$$
\frac{\partial \mathbf{u}}{\partial t}-\frac{\nu}{g}(\nabla \cdot(g \nabla)) \mathbf{u}+\nu\left(\frac{\nabla g}{g} \cdot \nabla\right) \mathbf{u}+(\mathbf{u} \cdot \nabla) \mathbf{u}+\nabla p=\mathbf{f} .
$$

Roh [3] proved the existence of solutions for periodic boundary conditions as well as Dirichlet boundary conditions on bounded domains. Global attractors are also discussed for suitable $g$. For these results, we need the smoothness of $g$ and the smallness of $\|\nabla g\|_{\infty}$. Refer to [3] for the details on $g$-Navier-Stokes equations.

In this paper, we prove the existence of the solutions for the $g$-Navier-Stokes equation (1.3)-(1.4) on the whole domain $\mathbf{R}^{n}$.

In section 2, we give a short introduction for the $g$-Navier-Stokes equations. In section 3, we review the solution space for the equations. In section 4, we consider the nonlinear term and perturbation term. In section 5, we review the compactness theorem in [5]. In section 6, we prove our main result about the existence. In section 7 , we show the solution obtained in section 6 is unique.

\section{Short Introduction of $g$-NAVIER-StOKes Equations}

Let ${ }_{3}={ }_{2} \times[0,1]$. Let $\mathbf{U}, \mathbf{V}$ be functions of $y=\left(y_{1}, y_{2}, y_{3}\right) \in g$ where $\left(y_{1}, y_{2}\right) \in 2$ and $0 \cdot y_{3} \cdot g\left(y_{1}, y_{2}\right)$. Then the change of variables

$$
y_{1}=x_{1}, y_{2}=x_{2}, y_{3}=x_{3} g\left(x_{1}, x_{2}\right)
$$

maps 3 onto $g$. The standard $3 D$ Navier-Stokes equations have the form

$$
\begin{array}{r}
\frac{\partial \mathbf{U}}{\partial t}-\nu \Delta \mathbf{U}+(\mathbf{U} \cdot \nabla) \mathbf{U}+\nabla \Phi=\mathbf{F} \\
\nabla \cdot \mathbf{U}=0
\end{array}
$$

on $g$. We assume that $\mathbf{U}$ satisfy the boundary condition

$$
\mathbf{U} \cdot \mathbf{n}=0 \quad \text { on } \quad \partial_{\text {top }} \quad g \cup \partial_{\text {bottom }} g
$$


where

$$
\begin{aligned}
\partial_{\text {top }} \quad g & =\left\{\left(y_{1}, y_{2}, y_{3}\right) \in \quad g: y_{3}=g\left(y_{1}, y_{2}\right)\right\} \\
\partial_{\text {bottom }} g & =\left\{\left(y_{1}, y_{2}, y_{3}\right) \in \quad g: y_{3}=0\right\} .
\end{aligned}
$$

Let $\mathbf{u}\left(x_{1}, x_{2}, x_{3}\right)=\mathbf{U}\left(y_{1}, y_{2}, y_{3}\right)$, where $\mathbf{x}=\left(x_{1}, x_{2}, x_{3}\right)$ and $\mathbf{y}=\left(y_{1}, y_{2}, y_{3}\right)$ satisfy (2.1).

Now we define $\mathbf{v}=\left(\mathbf{v}_{1}, \mathbf{v}_{2}\right)$ as $\mathbf{v}_{i}=\mathbf{v}_{i}\left(x_{1}, x_{2}\right)=\int_{0}^{1} \mathbf{u}_{i}\left(x_{1}, x_{2}, x_{3}\right) d x_{3}=\frac{1}{g\left(y_{1}, y_{2}\right)} \int_{0}^{g\left(y_{1}, y_{2}\right)} \mathbf{u}_{i}\left(y_{1}, y_{2}, y_{3}\right) d y_{3}$, for $i=1,2$ and we get the following proposition.

Proposition 2.1. Assume that $\nabla \cdot \mathbf{U}=0$ in $g$ and that (??) is valid. Then one has

$$
\nabla_{2} \cdot(g \mathbf{v})=\frac{\partial\left(g \mathbf{v}_{1}\right)}{\partial x_{1}}+\frac{\partial\left(g \mathbf{v}_{2}\right)}{\partial x_{2}}=\nabla g \cdot \mathbf{v}+g\left(\nabla_{2} \cdot \mathbf{v}\right)=0
$$

where $\nabla_{2}=\left(\frac{\partial}{\partial x_{1}}, \frac{\partial}{\partial x_{2}}\right)$ and $\nabla g=\left(\frac{\partial g}{\partial x_{1}}, \frac{\partial g}{\partial x_{2}}\right)$.

Proof. See Roh [3]

Next, we need the following assumption.

Assumption 1. $g(\mathbf{x}) \in C^{2}\left(\mathbf{R}^{n}\right)$ and $0<m \cdot g(\mathbf{x}) \cdot M$, for all $\mathbf{x} \in \mathbf{R}^{n}$, where $m=m(g)$ and $M=M(g)$. We also assume

$$
\|\nabla g\|_{\infty}=\sup _{(x, y) \in \mathbf{R}^{n}}|\nabla g(x, y)|<+\infty .
$$

\section{Functional Spaces}

We consider the physical domain $=\mathbf{R}^{n}$ for $n=2,3$. We denote by $L^{2}(, g)$ the space with the scalar product and the norm given by

$$
\langle\mathbf{u}, \mathbf{v}\rangle_{g}=\int(\mathbf{u} \cdot \mathbf{v}) g d \mathbf{x} \quad \text { and } \quad|\mathbf{u}|^{2}=\langle\mathbf{u}, \mathbf{u}\rangle_{\mathbf{g}}
$$

where $\mathbf{x}=\left(x_{1}, \cdots, x_{n}\right)$. Similarly, we will use the space $H^{1}(, g)$ with the norm by

$$
\|\mathbf{u}\|_{H^{1}(\quad, g)}=\left[\langle\mathbf{u}, \mathbf{u}\rangle_{g}+\sum_{i=1}^{n}\left\langle\partial_{i} \mathbf{u}, \partial_{i} \mathbf{u}\right\rangle_{g}\right]^{\frac{1}{2}},
$$


where $\frac{\partial \mathbf{u}}{\partial x_{i}}=\partial_{i} \mathbf{u}$.

Remark 1. Since $0<m \cdot g(\mathbf{x}) \cdot M$ for all $\mathbf{x} \in \mathbf{R}^{n}$, and $g$ is smooth, $|\mathbf{u}|_{L^{2}\left(\mathbf{R}^{n}\right)}$ is equivalent to $|\mathbf{u}|_{g}$ as well as $\|\mathbf{u}\|_{H^{1}\left(\mathbf{R}^{n}\right)}$ is equivalent to $\|\mathbf{u}\|_{H^{1}\left(\mathbf{R}^{n}, g\right)}$.

Let $\mathcal{D}\left(\mathbf{R}^{n}\right)$ be the space of $C^{\infty}$ functions with compact support contained in $\mathbf{R}^{n}$. The closure of $\mathcal{D}\left(\mathbf{R}^{n}\right)$ in $W^{m, p}\left(\mathbf{R}^{n}\right)$ is denoted by $W_{0}^{m, p}\left(\mathbf{R}^{n}\right)\left(H_{0}^{m}\left(\mathbf{R}^{n}\right)\right.$ when $p=2$ ).

For the mathematical setting, we define the spaces as the followings,

$$
\begin{aligned}
\mathcal{V} & =\left\{\mathbf{u} \in \mathcal{D}\left(\mathbf{R}^{n}\right): \nabla \cdot(g \mathbf{u})=0\right\} \\
H_{g} & =\text { the closure of } \mathcal{V} \text { in } L^{2}\left(\mathbf{R}^{n}\right) \\
V_{g} & =\text { the closure of } \mathcal{V} \text { in } H_{0}^{1}\left(\mathbf{R}^{n}\right),
\end{aligned}
$$

where $H_{g}$ are endowed with the scalar product and the norm in $L^{2}\left(\mathbf{R}^{n}, g\right)$, and $V_{g}$ are endowed with the scalar product and the norm in $H^{1}\left(\mathbf{R}^{n}, g\right)$. The space $V_{g}$ is contained in $H_{g}$, is dense in $H_{g}$, and the injection is continuous. Let $H_{g}^{\prime}$ and $V_{g}^{\prime}$ denote the dual spaces of $H_{g}$ and $V_{g}$, and let $i$ denote the injection mapping from $V_{g}$ into $H_{g}$. The adjoint operator $i^{\prime}$ is linear continuous from $H^{\prime}$ into $V_{g}^{\prime}$, and is one to one since $i\left(V_{g}\right)=V_{g}$ is dense in $H_{g}$ and $i^{\prime}\left(H_{g}^{\prime}\right)$ is dense in $V_{g}^{\prime}$ since $i$ is one to one. Therefore $H_{g}^{\prime}$ can be indentified with a dense subspace of $V_{g}^{\prime}$. Moreover, by the Riesz representation theorem, we can identify $H_{g}$ and $H_{g}^{\prime}$, and we arrive at the inclusions

$$
V_{g} \subset H_{g}=H_{g}^{\prime} \subset V_{g}^{\prime},
$$

where each space is dense in the following one and the injections are continuous. So we note that the scalar product in $H_{g}$ of $\mathbf{f} \in H_{g}$ and $\mathbf{u} \in V_{g}$ is the same as the scalar product of $\mathbf{f}$ and $\mathbf{u}$ in the duality between $V_{g}^{\prime}$ and $V_{g}$,

$$
\langle\mathbf{f}, \mathbf{u}\rangle_{g}=(\mathbf{f}, \mathbf{u}), \quad \forall \mathbf{f} \in H_{g}, \quad \forall \mathbf{u} \in V_{g} .
$$

For each $\mathbf{u}$ in $V_{g}$, the form

$$
\mathbf{v} \in V_{g} \rightarrow((\mathbf{u}, \mathbf{v}))_{g} \in \mathbf{R}
$$

is linear and continuous on $V_{g}$; therefore, there exist an element of $V_{g}^{\prime}$ which we denote by $A \mathbf{u}$ such that

$$
\langle A \mathbf{u}, \mathbf{v}\rangle_{g}=((\mathbf{u}, \mathbf{v}))_{g}, \quad \forall \mathbf{v} \in V_{g}
$$

where

$$
((\mathbf{u}, \mathbf{v}))_{g}=\sum_{i=1}^{n}\left\langle D_{i} \mathbf{u}, D_{i} \mathbf{v}\right\rangle_{g}
$$

Also, we denote

$$
\|\mathbf{u}\|^{2}=((\mathbf{u}, \mathbf{u}))_{g}=\sum_{i=1}^{n}\left\langle D_{i} \mathbf{u}, D_{i} \mathbf{u}\right\rangle_{g}
$$


Therefore, we have

$$
\|\mathbf{u}\|_{V_{g}}^{2}=|\mathbf{u}|^{2}+\|\mathbf{u}\|^{2}
$$

where $\|\mathbf{u}\|_{H_{g}}=|\mathbf{u}|$.

Problem 1. Given $\mathbf{f} \in L^{2}\left(0, T ; V_{g}^{\prime}\right)$ and $\mathbf{u}_{0} \in H_{g}$, to find $\mathbf{u}$ satisfying

$$
\begin{aligned}
& \mathbf{u} \in L^{2}\left(0, T ; V_{g}\right), \quad \mathbf{u}^{\prime} \in L^{2}\left(0, T ; V_{g}^{\prime}\right), \\
& \mathbf{u}^{\prime}+\nu A \mathbf{u}=\mathbf{f}, \quad \text { on }(0, T), \\
& \mathbf{u}(0)=\mathbf{u}_{0} .
\end{aligned}
$$

Lemma 3.1. Problem 1 has unique solution $\mathbf{u}$ and moreover $\mathbf{u} \in C\left([0, T] ; H_{g}\right)$.

Proof. One can prove by similar method in Chapter 3, [5].

Remark 2. Assuming that $\mathbf{f}, \mathbf{u}_{0}$ are sufficiently smooth, we can obtain as much regularity as desired for $\mathbf{u}$ and $p$. For given $\mathbf{f} \in L^{2}\left(0, T ; H_{g}\right)$ and $\mathbf{u}_{0} \in V_{g}$, one can obtain that

$$
\begin{aligned}
& \mathbf{u} \in L^{2}\left(0, T ; H^{2}()\right), \\
& \mathbf{u}^{\prime} \in L^{2}\left(0, T ; H_{g}\right), \text { and } p \in L^{2}\left(0, T ; H^{1}()\right) .
\end{aligned}
$$

For our problem, one should note that

$$
-\frac{1}{g}(\nabla \cdot g \nabla) \mathbf{u}=-\Delta \mathbf{u}-\left(\frac{\nabla g}{g} \cdot \nabla\right) \mathbf{u}
$$

Therefore, one obtains

$$
\langle-\Delta \mathbf{u}, \mathbf{v}\rangle_{g}=((\mathbf{u}, \mathbf{v}))_{g}+\left\langle\left(\frac{\nabla g}{g} \cdot \nabla\right) \mathbf{u}, \mathbf{v}\right\rangle_{g}=\langle A \mathbf{u}, \mathbf{v}\rangle_{g}+\left\langle\left(\frac{\nabla g}{g} \cdot \nabla\right) \mathbf{u}, \mathbf{v}\right\rangle_{g},
$$

for $\mathbf{u}, \mathbf{v} \in V_{g}$.

\section{Nonlinear and Perturbation Terms}

We define the trilinear form

$$
b(\mathbf{u}, \mathbf{v}, \mathbf{w})=\sum_{i, j=1}^{n} \int_{R^{n}} \mathbf{u}_{i}\left(D_{i} \mathbf{v}_{j}\right) \mathbf{w}_{j} g d x,
$$

where $\mathbf{u}, \mathbf{v}, \mathbf{w}$ lie in appropriate subspaces of $L^{2}\left(R^{n}, g\right)$ and $D_{i}=\frac{\partial}{\partial x_{i}}$. Since $\nabla \cdot g \mathbf{u}=\sum_{i} D_{i}\left(g \mathbf{u}_{i}\right)=0$, for $\mathbf{u} \in H_{g}$, one obtains 


$$
\begin{aligned}
b(\mathbf{u}, \mathbf{v}, \mathbf{w}) & =\sum_{i, j=1}^{n} \int_{R^{n}} \mathbf{u}_{i}\left(D_{i} \mathbf{v}_{j}\right) \mathbf{w}_{j} g d x \\
& =-\sum_{i, j=1}^{n} \int_{R^{n}} D_{i}\left(g \mathbf{u}_{i}\right) \mathbf{v}_{j} \mathbf{w}_{j} d x-\sum_{i, j=1}^{n} \int_{R^{n}} g \mathbf{u}_{i} \mathbf{v}_{j}\left(D_{i} \mathbf{w}_{j}\right) d x \\
& =-\sum_{i, j=1}^{n} \int_{R^{n}} g \mathbf{u}_{i} \mathbf{v}_{j}\left(D_{i} \mathbf{w}_{j}\right) d x=-b(\mathbf{u}, \mathbf{w}, \mathbf{v}),
\end{aligned}
$$

for sufficient smooth functions $\mathbf{u}, \mathbf{v}, \mathbf{w} \in H_{g}$. Therefore $b(\mathbf{u}, \mathbf{v}, \mathbf{w})=-b(\mathbf{u}, \mathbf{w}, \mathbf{v})$ and $b(\mathbf{u}, \mathbf{v}, \mathbf{v})=0$, for smooth functions $\mathbf{u}, \mathbf{v}, \mathbf{w} \in H_{g}$.

For $\mathbf{u}, \mathbf{v}$ in $V_{g}$, we denote by $B(\mathbf{u}, \mathbf{v})$ the element of $V_{g}^{\prime}$ defined by

$$
\langle B(\mathbf{u}, \mathbf{v}), \mathbf{w}\rangle_{g}=b(\mathbf{u}, \mathbf{v}, \mathbf{w}), \quad \forall \mathbf{w} \in V_{g},
$$

and we set

$$
B(\mathbf{u})=B(\mathbf{u}, \mathbf{u}) \in V_{g}^{\prime}, \quad \forall \mathbf{u} \in V_{g} .
$$

Before we estimate the nonlinear term $B(\mathbf{u})$, let us look at the useful inequalities.

Lemma 4.1 If $n=2$, then we have

$$
\|\mathbf{u}\|_{L^{4}\left(\mathbf{R}^{2}, g\right)} \cdot \quad c|\mathbf{u}|^{\frac{1}{2}}\|\mathbf{u}\|^{\frac{1}{2}}, \quad \forall \mathbf{u} \in H^{1}\left(\mathbf{R}^{2}, g\right) .
$$

and if $n=3$, then we have

$$
\|\mathbf{u}\|_{L^{4}\left(\mathbf{R}^{3}, g\right)} \cdot \quad c|\mathbf{u}|^{\frac{1}{4}}\|\mathbf{u}\|^{\frac{3}{4}}, \quad \forall \mathbf{u} \in H^{1}\left(\mathbf{R}^{3}, g\right) .
$$

Proof. One can easily see by the equivalence of the norms. by

Lemma 4.2 We assume that $\mathbf{u} \in L^{2}\left(0, T ; V_{g}\right)$. Then the function Bu defined

$$
\langle B \mathbf{u}(t), \mathbf{v}\rangle_{g}=b(\mathbf{u}(t), \mathbf{u}(t), \mathbf{v}), \forall \mathbf{u} \in V_{g}, \text { a.e. } t \in[0, T],
$$

belongs to $L^{1}\left(0, T ; V_{g}^{\prime}\right)$. Moreover, the function $C \mathbf{u}$ defined by

$$
\langle C \mathbf{u}(t), \mathbf{v}\rangle_{g}=\left\langle\left(\frac{\nabla g}{g} \cdot \nabla\right) \mathbf{u}, \mathbf{v}\right\rangle_{g}=\sum_{i, j=1}^{2} \int_{R^{n}} \frac{D_{i} g}{g}\left(D_{i} \mathbf{u}_{j}\right) \mathbf{v}_{j} g d \mathbf{x}=b\left(\frac{\nabla g}{g}, \mathbf{u}, \mathbf{v}\right),
$$

for all $\mathbf{v} \in V_{g}$, belong to $L^{2}\left(0, T ; H_{g}\right)$, and hence belong to $L^{2}\left(0, T ; V_{g}^{\prime}\right)$. 
Proof. One can easily check by the previous lemma that for almost all $t$, $B \mathbf{u}(t) \in V_{g}^{\prime}$. For $\mathbf{u}, \mathbf{v} \in V_{g}$, one has

$$
\begin{aligned}
\left|\langle B(\mathbf{u}), \mathbf{v}\rangle_{g}\right| & =|b(\mathbf{u}, \mathbf{u}, \mathbf{v})| \\
& =\left|\int_{\mathbf{R}^{n}} \sum_{i, j=1}^{n} \mathbf{u}_{i}\left(D_{i} \mathbf{u}_{j}\right) \mathbf{v}_{j} g d \mathbf{x}\right| \\
& =\left|\int_{\mathbf{R}^{n}} \sum_{i, j=1}^{n} \mathbf{u}_{i}\left(D_{i} \mathbf{v}_{j}\right) \mathbf{u}_{j} g d \mathbf{x}\right| \\
& \cdot c\|\mathbf{v}\|\|\mathbf{u}\|_{L^{4}\left(\mathbf{R}^{n}, g\right)}^{2} \cdot c\|\mathbf{v}\|_{V_{g}}\|\mathbf{u}\|_{L^{4}\left(\mathbf{R}^{n}, g\right)}^{2} .
\end{aligned}
$$

So, if $n=2$, then

$$
\|B(\mathbf{u})\|_{V_{g}^{\prime}} \cdot c\|\mathbf{u}\|_{L^{4}\left(\mathbf{R}^{2}, g\right)}^{2} \cdot c|\mathbf{u}|\|\mathbf{u}\| .
$$

Also, if $n=3$, then

$$
\|B(\mathbf{u})\|_{V_{g}^{\prime}} \cdot c\|\mathbf{u}\|_{L^{4}\left(\mathbf{R}^{2}, g\right)}^{2} \cdot c|\mathbf{u}|^{\frac{1}{2}}\|\mathbf{u}\|^{\frac{3}{2}} .
$$

Hence, for $n=2,3$, one has that

$$
\|B \mathbf{u}\|_{V_{g}^{\prime}} \cdot c\|\mathbf{u}\|_{V_{g}}^{2}, \forall \mathbf{u} \in V_{g}
$$

for some constant $c$. Hence, we obtain

$$
\int_{0}^{T}\|B \mathbf{u}\|_{V_{g}^{\prime}} d t \cdot \quad c \int_{0}^{T}\|\mathbf{u}(t)\|_{V_{g}}^{2} d t<+\infty
$$

which implies that $B \mathbf{u}$ belong to $L^{1}\left(0, T ; V_{g}^{\prime}\right)$.

Next, for the estimate of $C \mathbf{u}$, we have

$$
\begin{aligned}
|\langle C \mathbf{u}, \mathbf{v}\rangle| & =\left|\sum_{i, j=1}^{n} \int_{\mathbf{R}^{n}} \frac{D_{i} g}{g}\left(D_{i} \mathbf{u}_{j}\right) \mathbf{v}_{j} g d \mathbf{x}\right| \\
& \cdot c\|\nabla g\|_{\infty}\|\mathbf{u}\||\mathbf{v}| .
\end{aligned}
$$

So, one obtains

$$
|C \mathbf{u}(t)| \cdot c\|\nabla g\|_{\infty}\|\mathbf{u}\|
$$

Hence, we have

$$
\int_{0}^{T}|C \mathbf{u}(t)|^{2} d t \cdot c\|\nabla g\|_{\infty}^{2} \int_{0}^{T}\|\mathbf{u}\|^{2} d t \cdot c\|\nabla g\|_{\infty}^{2} \int_{0}^{T}\|\mathbf{u}\|_{V_{g}}^{2} d t<+\infty
$$

which implies that $C \mathbf{u}(t)$ belong to $L^{2}\left(0, T ; H_{g}\right)$. 


\section{COMPACTNESS}

The following two propositions are stated in [5].

Proposition 5.1. Let $X_{0}, X$ and $X_{1}$ be three Banach spaces such that

$$
X_{0} \subset X \subset X_{1},
$$

the injection of $X$ into $X_{1}$ being continuous, and the injection of $X_{0}$ into $X$ is compact. Then for every $\eta>0$, there exist some constant $c_{\eta}$ depending on $\eta$ (and on the spaces $X_{0}, X, X_{1}$ ) such that:

$$
\|\mathbf{v}\|_{X} \cdot \eta\|\mathbf{v}\|_{X_{0}}+c_{\eta}\|\mathbf{v}\|_{X_{1}}, \quad \forall \mathbf{v} \in X_{0} .
$$

Now, we assume that $X_{0}, X, X_{1}$, are Hilbert spaces with

$$
X_{0} \subset X \subset X_{1}
$$

the injections being continuous and

$$
\text { the injection of } X_{0} \text { into } X \text { is compact. }
$$

If $\mathbf{v}$ is a function from $\mathbf{R}$ into $X_{1}$, we denote by $\hat{\mathbf{v}}$ its Fourier transform

$$
\hat{\mathbf{v}}(\tau)=\int_{-\infty}^{\infty} e^{-2 i \pi t \tau} \mathbf{v}(t) d t
$$

The derivative in $t$ of order $\gamma$ of $\mathbf{v}$ is the inverse Fourier transform of $(2 i \pi \tau)^{\gamma} \hat{\mathbf{v}}$ or

$$
\widehat{D_{t}^{\gamma} \mathbf{v}}(\tau)=(2 i \pi \tau)^{\gamma} \hat{\mathbf{v}}(\tau) \text {. }
$$

For given $\gamma>0$, we define the space

$$
\mathcal{H}^{\gamma}\left(\mathbf{R} ; X_{0}, X_{1}\right)=\left\{\mathbf{v} \in L^{2}\left(\mathbf{R} ; X_{0}\right), D_{t}^{\gamma} \mathbf{v} \in L^{2}\left(\mathbf{R} ; X_{1}\right)\right\} .
$$

This is a Hilbert space for the norm,

$$
\|\mathbf{v}\|_{\mathcal{H}^{\gamma}\left(\mathbf{R}, X_{0}, X_{1}\right)}=\left\{\|\mathbf{v}\|_{L^{2}\left(\mathbf{R} ; X_{0}\right)}^{2}+\left\||\tau|^{\gamma} \hat{\mathbf{v}}\right\|_{L^{2}\left(\mathbf{R} ; X_{1}\right)}^{2}\right\}^{\frac{1}{2}} .
$$

We also define the subspace $\mathcal{H}_{K}^{\gamma}$ of $\mathcal{H}^{\gamma}$, for any set $K \subset \mathbf{R}$, as

$$
\mathcal{H}_{K}^{\gamma}\left(\mathbf{R} ; X_{0}, X_{1}\right)=\left\{\mathbf{u} \in \mathcal{H}^{\gamma}\left(\mathbf{R} ; X_{0}, X_{1}\right), \text { support } \mathbf{u} \subset K\right\} .
$$

Proposition 5.2. Let us assume that $X_{0}, X, X_{1}$ are Hilbert spaces which satisfy (5.1) and (5.2).

Then for any bounded set $K$ and any $\gamma>0$, the injection of $H_{K}^{\gamma}\left(\mathbf{R} ; X_{0}, X_{1}\right)$ into $L^{2}(\mathbf{R}, X)$ is compact. 
Remark 3. Let us recall the mathematical spaces for our problem. For the mathematical setting, we defined the spaces as the followings,

$$
\begin{aligned}
\mathcal{V} & =\left\{\mathbf{u} \in \mathcal{D}\left(\mathbf{R}^{n}\right), \nabla \cdot(g \mathbf{u})=0\right\} \\
H_{g} & =\text { the closure of } \mathcal{V} \text { in } L^{2}\left(\mathbf{R}^{n}\right) \\
V_{g} & =\text { the closure of } \mathcal{V} \text { in } H_{0}^{1}\left(\mathbf{R}^{n}\right),
\end{aligned}
$$

where $H_{g}$ are endowed with the scalar product and the norm in $L^{2}\left(\mathbf{R}^{n}, g\right)$, and $V_{g}$ are endowed with the scalar product and the norm in $H^{1}\left(\mathbf{R}^{n}, g\right)$. The space $V_{g}$ is contained in $H_{g}$, is dense in $H_{g}$, and the injection is continuous. But, the injection is not compact. So, we can not use the previous compactness theorem. Hence, to use the previous compactness theorem, we consider a bounded ball $\mathcal{Q}$ in $\mathbf{R}^{n}$ instead of $\mathbf{R}^{n}$ and

$$
\begin{aligned}
\mathcal{V} & =\{\mathbf{u} \in \mathcal{D}(\mathcal{Q}), \nabla \cdot(g \mathbf{u})=0\} \\
H_{g}(\mathcal{Q}) & =\text { the closure of } \mathcal{V} \text { in } L^{2}(\mathcal{Q}) \\
V_{g}(\mathcal{Q}) & =\text { the closure of } \mathcal{V} \text { in } H_{0}^{1}(\mathcal{Q}) .
\end{aligned}
$$

Then the space $V_{g}(\mathcal{Q})$ is contained in $H_{g}(\mathcal{Q})$, is dense in $H_{g}(\mathcal{Q})$, and the injection being continuous is compact. Therefore, we can use the previous compactness theorem and we have the following lemma.

Lemma 5.3. If $\mathbf{u}_{k}$ converges to $\mathbf{u}$ in $L^{2}\left(0, T ; V_{g}(\mathcal{Q})\right)$ weakly and $L^{2}\left(0, T ; H_{g}(\mathcal{Q})\right)$ strongly, then for any vector function $\mathbf{w}$ with components in $C_{0}^{1}(\mathcal{Q})$,

$$
\int_{0}^{T} b\left(\mathbf{u}_{k}(t), \mathbf{u}_{k}(t), \mathbf{w}(t)\right) d t \rightarrow \int_{0}^{T} b(\mathbf{u}(t), \mathbf{u}(t), \mathbf{w}(t)) d t .
$$

Proof. We note that

$$
\begin{aligned}
\int_{0}^{T} b\left(\mathbf{u}_{k}, \mathbf{u}_{k}, \mathbf{w}\right) d t & =-\int_{0}^{T} b\left(\mathbf{u}_{k}, \mathbf{w}, \mathbf{u}_{k}\right) \\
& =-\sum_{i, j=1}^{n} \int_{0}^{T} \int\left(\mathbf{u}_{k}\right)_{i}\left(D_{i} \mathbf{w}_{j}\right)\left(\mathbf{u}_{k}\right)_{j} g d \mathbf{x} d t .
\end{aligned}
$$

These integrals converge to

$$
-\sum_{i, j=1}^{n} \int_{0}^{T} \int \mathbf{u}_{i}\left(D_{i} \mathbf{w}_{j}\right) \mathbf{u}_{j} g d \mathbf{x} d t=-\int_{0}^{T} b(\mathbf{u}, \mathbf{w}, \mathbf{u}) d t=\int_{0}^{T} b_{g}(\mathbf{u}, \mathbf{u}, \mathbf{w}) d t,
$$

because $g$ is bounded function on $\mathbf{R}^{n}$ and $\mathbf{w} \in C_{0}^{1}(\mathcal{Q})$. 


\section{Proof of Existence}

The initial value problem of the $g$-Navier-Stokes equations is to find suitable vector function $\mathbf{u}$ and scalar function $p$ such that

$$
\mathbf{u}: \quad \times[0, T] \rightarrow \mathbf{R}^{n}, \quad p: \quad \times[0, T] \rightarrow \mathbf{R}
$$

satisfying

$$
\begin{aligned}
& \frac{\partial \mathbf{u}}{\partial t}-\nu \Delta \mathbf{u}+\sum_{i=1}^{n} \mathbf{u}_{i} D_{i} \mathbf{u}+\nabla p=\mathbf{f} \text { in } \quad \times(0, T), \\
& \frac{1}{g}(\nabla \cdot(g \mathbf{u}))=\nabla \cdot \mathbf{u}+\left(\frac{\nabla g}{g} \cdot \mathbf{u}\right)=0 \quad \text { in } \quad \times(0, T), \\
& \mathbf{u}(\mathbf{x}, 0)=\mathbf{u}_{0}(\mathbf{x}) \quad \text { in } \quad .
\end{aligned}
$$

Problem 2. For $\mathbf{f} \in L^{2}\left(0, T ; V_{g}^{\prime}\right)$ and $\mathbf{u}_{0} \in H_{g}$, to find $\mathbf{u} \in L^{2}\left(0, T ; V_{g}\right)$ satisfying

$$
\begin{aligned}
\frac{d}{d t}(\mathbf{u}, \mathbf{v})_{g}+\nu((\mathbf{u}, \mathbf{v}))_{g} & +b(\mathbf{u}, \mathbf{u}, \mathbf{v}) \\
& =\langle\mathbf{f}, \mathbf{v}\rangle_{g}-\left\langle\left(\frac{\nabla g}{g} \cdot \nabla\right) \mathbf{u}, \mathbf{v}\right\rangle_{g} \quad \forall \mathbf{v} \in V_{g}
\end{aligned}
$$

and

$$
\mathbf{u}(0)=\mathbf{u}_{0} .
$$

If $\mathbf{u} \in L^{2}\left(0, T ; V_{g}\right)$ satisfies the equation (6.1), then by (3.1), (3.2) and lemma 4.2 , one can write the equation (6.1) as

$$
\frac{d}{d t}\langle\mathbf{u}, \mathbf{v}\rangle=\langle\mathbf{f}-\nu A \mathbf{u}-B \mathbf{u}-C \mathbf{u}, \mathbf{v}\rangle, \forall \mathbf{v} \in V_{g} .
$$

One note that since $A \mathbf{u}$ belong to $L^{2}\left(0, T ; V_{g}^{\prime}\right)$, the function $\mathbf{f}-\nu A \mathbf{u}-B \mathbf{u}-C \mathbf{u}$ belong to $L^{1}\left(0, T ; V_{g}^{\prime}\right)$.

Theorem 6.1. Assume that $\mathbf{f} \in L^{2}\left(0, T ; V_{g}^{\prime}\right)$ and $\mathbf{u}_{0} \in H_{g}$. Then there exist at least one solution $\mathbf{u}$ of problem 2. Moreover,

$$
\mathbf{u} \in L^{\infty}\left(0, T ; H_{g}\right)
$$

and $\mathbf{u}$ is weakly continuous from $[0, T]$ into $H_{g}$.

Proof. We apply the Galerkin procedure. Since $V_{g}$ is seperable and $\mathcal{V}$ is dense in $V_{g}$, there exists a sequence $\mathbf{w}_{1}, \ldots ., \mathbf{w}_{m}, \ldots$ of elelments of $\mathcal{V}$, which is free and total in $V_{g}$. For each $m$ we define an approximate solution $\mathbf{u}_{m}$ of equation (6.1) as

$$
\mathbf{u}_{m}=\sum_{i=1}^{m} \phi_{i m}(t) \mathbf{w}_{i}
$$


which satisfies

$$
\begin{aligned}
\left(\mathbf{u}_{m}^{\prime}(t), \mathbf{w}_{j}\right)+ & \nu\left(\left(\mathbf{u}_{m}(t), \mathbf{w}_{j}\right)\right)_{g}-b\left(\frac{\nabla g}{g}, \mathbf{u}_{m}(t), \mathbf{w}_{j}\right) \\
& +b\left(\mathbf{u}_{m}(t), \mathbf{u}_{m}(t), \mathbf{w}_{j}\right)=\left\langle\mathbf{f}(t), \mathbf{w}_{j}\right\rangle_{g},
\end{aligned}
$$

for $t \in[0, T], j=1, \ldots, m$, and $\mathbf{u}_{m}(0)=\mathbf{u}_{0 m}$, where $\mathbf{u}_{0 m}$ is the orthogonal projection in $H_{g}$ of $\mathbf{u}_{0}$ onto the space spanned by $\mathbf{w}_{1}, \ldots, \mathbf{w}_{m}$. Then one can get

$$
\begin{aligned}
& \sum_{i=1}^{m}\left(\mathbf{w}_{i}, \mathbf{w}_{j}\right) \phi_{i m}^{\prime}(t)+\nu \sum_{i=1}^{m}\left(\left(\mathbf{w}_{i}, \mathbf{w}_{j}\right)\right)_{g} \phi_{i m}(t) \\
& +\sum_{i}^{m} b\left(\frac{\nabla g}{g}, \mathbf{w}_{i}, \mathbf{w}_{j}\right) \phi_{i m}(t)+\sum_{i, l=1}^{m} b\left(\mathbf{w}_{i}, \mathbf{w}_{l}, \mathbf{w}_{j}\right) \phi_{i m}(t) \phi_{l m}(t) \\
& =\left\langle\mathbf{f}(t), \mathbf{w}_{j}\right\rangle_{g} .
\end{aligned}
$$

Inverting the nonsingular matrix with elements $\left\langle\mathbf{w}_{i}, \mathbf{w}_{j}\right\rangle_{g}, 1 \cdot i, j \cdot m$, we can write the differential equations in the usual form

$$
\phi_{i m}^{\prime}(t)+\sum_{j=1}^{m} \alpha_{i j} \phi_{j m}(t)+\sum_{j, k=1}^{m} \alpha_{i j k} \phi_{j m}(t) \phi_{k m}(t)=\sum_{j=1}^{m} \beta_{i j}\left\langle\mathbf{f}(t), \mathbf{w}_{j}\right\rangle_{g},
$$

where $\alpha_{i j}, \alpha_{i j k}, \beta_{i j} \in \mathbf{R}$. Let

$$
\phi_{i m}(0)=\text { the } i^{t h} \text { component of } \mathbf{u}_{0 m} .
$$

The nonlinear ordinary differential system (6.4) with the initial condition (6.5) has a maximal solution defined on some interval $\left[0, t_{m}\right]$. If $t_{m}<T$, then $\left|\mathbf{u}_{m}(t)\right|$ must tend to $+\infty$ as $t \rightarrow t_{m}$; the a priori estimates we shall prove later show that this does not happen and therefore $t_{m}=T$. To do that, we need several estimates.

(i) We multiply (6.3) by $\phi_{j m}(t)$ and add these equations for $j=1, \ldots, m$ to get

$$
\left.\left(\mathbf{u}_{m}^{\prime}(t), \mathbf{u}_{m}(t)\right)+\nu\left\|\mathbf{u}_{m}(t)\right\|^{2}=\left\langle\mathbf{f}(t), \mathbf{u}_{m}(t)\right\rangle_{g}-b\left(\frac{\nabla g}{g} \cdot \nabla\right) \mathbf{u}_{m}(t), \mathbf{u}_{m}(t)\right) .
$$

Then we write

$$
\begin{aligned}
\frac{d}{d t}\left|\mathbf{u}_{m}(t)\right|^{2}+ & \left.2 \nu\left\|\mathbf{u}_{m}(t)\right\|^{2}=2\left\langle\mathbf{f}(t), \mathbf{u}_{m}(t)\right\rangle_{g}+2 b\left(\frac{\nabla g}{g} \cdot \nabla\right) \mathbf{u}_{m}(t), \mathbf{u}_{m}(t)\right) \\
\cdot & 2\|\mathbf{f}(t)\|_{V^{\prime}}\left\|\mathbf{u}_{m}(t)\right\|_{V}+\frac{2}{m}|\nabla g|_{\infty}\left|\mathbf{u}_{m}(t)\right|\left\|\mathbf{u}_{m}(t)\right\|+\left\|\mathbf{u}_{m}\right\|^{2}, \\
\cdot & \nu\left\|\mathbf{u}_{m}(t)\right\|^{2}+\frac{8}{\nu}\|\mathbf{f}(t)\|_{V^{\prime}}^{2}+\frac{2}{\nu m^{2}}|\nabla g|_{\infty}^{2}\left|\mathbf{u}_{m}(t)\right|^{2}+\nu\left|\mathbf{u}_{m}\right|^{2},
\end{aligned}
$$

so that

$$
\frac{d}{d t}\left|\mathbf{u}_{m}(t)\right|^{2}+\nu\left\|\mathbf{u}_{m}(t)\right\|^{2} \cdot \frac{8}{\nu}\|\mathbf{f}(t)\|_{V^{\prime}}^{2}+\alpha\left|\mathbf{u}_{m}(t)\right|^{2},
$$


where $\alpha=\frac{2}{\nu m^{2}}|\nabla g|_{\infty}^{2}+\nu$.

Hence, one obtains

$$
\frac{d}{d t}\left|\mathbf{u}_{m}(t)\right|^{2} \cdot \alpha\left|\mathbf{u}_{m}(t)\right|^{2}+\frac{8}{\nu}\|\mathbf{f}(t)\|_{V^{\prime}}^{2}
$$

where $\alpha=\frac{2}{\nu m^{2}}|\nabla g|_{\infty}^{2}$. So, by the usual method of the Gronwall inequality, we have

$$
\left|\mathbf{u}_{m}(t)\right|^{2} \cdot e^{\alpha t}\left(\left|\mathbf{u}_{m}(0)\right|^{2}+\frac{8}{\nu} \int_{0}^{t}|\mathbf{f}(s)|_{V_{g}^{\prime}}^{2} d s\right) .
$$

By the assumption the right side of the above inequality is uniformly bounded for $s \in[0, T]$ and $m$.

Hence

$$
\sup _{s \in[0, T]}\left|\mathbf{u}_{m}(s)\right|^{2} \cdot e^{\alpha T}\left(\left|\mathbf{u}_{m}(0)\right|^{2}+\frac{8}{\nu} \int_{0}^{T}|\mathbf{f}(s)|_{V_{g}^{\prime}}^{2} d s\right)
$$

which implies that

(6.7) the sequence $\mathbf{u}_{m}$ remains in a bounded set of $L^{\infty}\left(0, T ; H_{g}\right)$.

(ii) For the convenience, let us define

$$
K(T)=e^{\alpha T}\left(\left|\mathbf{u}_{m}(0)\right|^{2}+\frac{8}{\nu} \int_{0}^{T}|\mathbf{f}(s)|_{V_{g}^{\prime}}^{2} d s\right) .
$$

Now, we integrate (6.6) from 0 to $T$ to get

$$
\begin{aligned}
\left|\mathbf{u}_{m}(T)\right|^{2} & +\nu \int_{0}^{T}\left\|\mathbf{u}_{m}(t)\right\|^{2} d t \\
\cdot & \left|\mathbf{u}_{0 m}\right|^{2}+\frac{8}{\nu} \int_{0}^{T}\|\mathbf{f}(t)\|_{V^{\prime}}^{2} d t+\alpha \int_{0}^{T}\left|\mathbf{u}_{m}(t)\right|^{2} d t \\
\cdot & \left|\mathbf{u}_{0}\right|^{2}+\frac{8}{\nu} \int_{0}^{T}\|\mathbf{f}(t)\|_{V^{\prime}}^{2} d t+\alpha K(T) T .
\end{aligned}
$$

Therefore,

the sequence $\mathbf{u}_{m}$ remains in a bounded set of $L^{2}\left(0, T ; V_{g}\right)$.

(iii) Let $\tilde{\mathbf{u}}_{m}$ denote the function from $\mathbf{R}$ into $V_{g}$, which is equal to $\mathbf{u}_{m}$ on $[0, T]$ and to 0 on the complitement of this interval. The Fourier transform of $\tilde{\mathbf{u}}_{m}$ is denoted by $\hat{\mathbf{u}}_{m}$. Then, we want to show that there exist a positive constant $c$ and $\gamma$ such that

$$
\int_{-\infty}^{\infty}|\tau|^{2 \gamma}\left|\hat{\mathbf{u}}_{m}(\tau)\right|^{2} d \tau \cdot c
$$


So, since the sequence $\mathbf{u}_{m}$ remains in a bounded set of $L^{2}\left(0, T ; V_{g}\right)$, the sequence $\tilde{\mathbf{u}}_{m}$ remains in a bounded set of $\mathcal{H}^{\gamma}\left(\mathbf{R} ; V_{g}, H_{g}\right)$.

It is classical that since $\tilde{\mathbf{u}}_{m}$ has two discontinuities, at 0 and $T$, the distribution derivative of $\tilde{\mathbf{u}}_{m}$ is given by

$$
\frac{d}{d t} \tilde{\mathbf{u}}_{m}=\tilde{\phi}_{m}+\mathbf{u}_{m}(0) \delta_{0}-\mathbf{u}_{m}(T) \delta_{T},
$$

where $\delta_{0}$ and $\delta_{T}$ are the Dirac distributions at 0 and $T$, and $\phi_{m}=\mathbf{u}_{m}^{\prime}$ is the derivative of $\mathbf{u}_{m}$ on $[0, T]$. Therefore by (6.3), one obtains that

$$
\frac{d}{d t}\left\langle\tilde{\mathbf{u}}_{m}, \mathbf{w}_{j}\right\rangle_{g}=\left\langle\tilde{\mathbf{f}}_{m}, \mathbf{w}_{j}\right\rangle_{g}+\left\langle\mathbf{u}_{0 m}, \mathbf{w}_{j}\right\rangle_{g} \delta_{0}-\left\langle\mathbf{u}_{m}(T), \mathbf{w}_{j}\right\rangle_{g} \delta_{T}
$$

for $j=1, \ldots, m$, where $\delta_{0}, \delta_{T}$ are Dirac distributions at 0 and $T, \mathbf{f}_{m}=\mathbf{f}-\nu A \mathbf{u}_{m}-$ $B \mathbf{u}_{m}-C \mathbf{u}_{m}$, and $\tilde{\mathbf{f}}_{m}=\mathbf{f}_{m}$ on $[0, T], 0$ outside this interval. By the Fourier transform, (6.11) gives

$$
\begin{aligned}
2 i \pi \tau\left\langle\hat{\mathbf{u}}_{m}, \mathbf{w}_{j}\right\rangle_{g} & =\left\langle\hat{\mathbf{f}}_{m}, \mathbf{w}_{j}\right\rangle_{g}+\left\langle\mathbf{u}_{0 m}, \mathbf{w}_{j}\right\rangle_{g} \\
& -\left\langle\mathbf{u}_{m}(T), \mathbf{w}_{j}\right\rangle_{g} \exp (-2 i \pi T \tau),
\end{aligned}
$$

$\hat{\mathbf{u}}_{m}$ and $\hat{\mathbf{f}}_{m}$ denoting the Fourier transforms of $\tilde{\mathbf{u}}_{m}$ and $\tilde{\mathbf{f}}_{m}$ respectively. We multiply (6.12) by $\hat{\phi_{j m}}(\tau)$ (=Fourier transform of $\left.\hat{\phi_{j m}}\right)$ and add the resulting equations for $j=1, \ldots, m$; we get:

$$
\begin{aligned}
2 i \pi \tau\left|\hat{\mathbf{u}}_{m}(\tau)\right|^{2} & =\left\langle\hat{\mathbf{f}}_{m}(\tau), \hat{\mathbf{u}}_{m}(\tau)\right\rangle_{g}+\left\langle\mathbf{u}_{0 m}, \hat{\mathbf{u}}_{m}(\tau)\right\rangle_{g} \\
& -\left\langle\mathbf{u}_{m}(T), \hat{\mathbf{u}}_{m}(\tau)\right\rangle_{g} \exp (-2 i \pi T \tau) .
\end{aligned}
$$

Because of inequality (3.2), (4.2), (4.3) and (4.4) one obtains

$$
\int_{0}^{T}\left\|\mathbf{f}_{m}(t)\right\|_{V_{g}^{\prime}} d t \cdot \int_{0}^{T}\left(\|\mathbf{f}(t)\|_{V_{g}^{\prime}}+\nu\left\|\mathbf{u}_{m}(t)\right\|+c\|\nabla g\|_{\infty}\left\|\mathbf{u}_{m}\right\|+c\left\|\mathbf{u}_{m}(t)\right\|_{V_{g}}^{2}\right) d t .
$$

Therefore, $\mathbf{f}_{m}(t)$ belong to a bounded set in the space $L^{1}\left(0, T ; V_{g}^{\prime}\right)$. Hence,

$$
\sup _{\tau \in R}\left\|\hat{\mathbf{f}}_{m}(\tau)\right\|_{V_{g}^{\prime}} \cdot \text { constant, } \forall m .
$$

So, by using

$$
\left|\mathbf{u}_{m}(0)\right| \cdot K(T), \quad\left|\mathbf{u}_{m}(T)\right| \cdot K(T),
$$

we deduce from (6.12) that

$$
|\tau|\left|\hat{\mathbf{u}}_{m}(\tau)\right|^{2} \cdot c_{2}\left\|\hat{\mathbf{u}}_{m}(\tau)\right\|_{V_{g}}+c_{3}\left|\hat{\mathbf{u}}_{m}(\tau)\right|
$$


or

$$
|\tau|\left|\hat{\mathbf{u}}_{m}(\tau)\right|^{2} \cdot c_{4}\left\|\hat{\mathbf{u}}_{m}(\tau)\right\|_{V_{g}}
$$

For $\gamma$ fixed, $\gamma<\frac{1}{4}$, we observe that

$$
|\tau|^{2 \gamma} \cdot c_{5}(\gamma) \frac{1+|\tau|}{1+|\tau|^{1-2 \gamma}}, \quad \forall \tau \in R
$$

Thus, by (6.13), we have

$$
\begin{aligned}
\int_{-\infty}^{+\infty}|\tau|^{2 \gamma}\left|\hat{\mathbf{u}}_{m}(\tau)\right|^{2} d \tau \cdot & c_{5}(\gamma) \int_{-\infty}^{+\infty} \frac{1+|\tau|}{1+\left.|\tau|\right|^{1-2 \gamma}}\left|\hat{\mathbf{u}}_{m}(\tau)\right|^{2} d \tau \\
\cdot & c_{6} \int_{-\infty}^{+\infty} \frac{\left\|\hat{\mathbf{u}}_{m}(\tau)\right\|_{V_{g}}}{1+|\tau|^{1-2 \gamma}} d \tau+c_{7} \int_{-\infty}^{+\infty}\left\|\hat{\mathbf{u}}_{m}(\tau)\right\|_{V_{g}}^{2} d \tau
\end{aligned}
$$

Since $\mathbf{u}_{m} \in L^{2}\left(0, T ; V_{g}\right)$, by the Parseval equality

$$
\int_{-\infty}^{+\infty}\left\|\hat{\mathbf{u}}_{m}(\tau)\right\|_{V_{g}}^{2} d \tau<\text { constant }
$$

Also, by the Schwarz inequality and the Parseval equality, one obtains

$$
\int_{-\infty}^{+\infty} \frac{\left\|\hat{\mathbf{u}}_{m}(\tau)\right\|_{V_{g}}}{1+|\tau|^{1-2 \gamma}} d \tau \cdot\left(\int_{-\infty}^{+\infty} \frac{1}{\left(1+|\tau|^{1-2 \gamma}\right)^{2}} d \tau\right)^{\frac{1}{2}}\left(\int_{-\infty}^{+\infty}\left\|\mathbf{u}_{m}(t)\right\|_{V_{g}}^{2} d t\right)^{\frac{1}{2}}
$$

which is finite since $\gamma<\frac{1}{4}$. So, the proof of (6.9) is achieved and $\mathbf{u}_{m} \in$ $\mathcal{H}^{\gamma}\left(\mathbf{R} ; V_{g}, H_{g}\right)$.

Therefore, so far, we obtained that $\mathbf{u}_{m}$ remains in a bounded set of $L^{\infty}\left(0, T ; H_{g}\right)$, $L^{2}\left(0, T ; V_{g}\right)$ and $\mathcal{H}^{\gamma}\left(\mathbf{R} ; V_{g}, H_{g}\right)$.

The estimates (6.7) and (6.8) enable us to assert the existence of an element $\mathbf{u} \in L^{2}\left(0, T ; V_{g}\right) \cap L^{\infty}\left(0, T ; H_{g}\right)$ and a sub-sequence $\mathbf{u}_{m^{\prime}}$ such that

$$
\mathbf{u}_{m^{\prime}} \rightarrow \mathbf{u} \text { in } L^{2}\left(0, T ; V_{g}\right) \text { weakly }
$$

and

$$
\mathbf{u}_{m^{\prime}} \rightarrow \mathbf{u} \text { in } L^{\infty}\left(0, T ; H_{g}\right) \text { weak-star }
$$

as $m^{\prime} \rightarrow \infty$. For any ball $\mathcal{Q}$ included in $\mathbf{R}^{n}$, the injection of $V_{g}(\mathcal{Q})$ into $H_{g}(\mathcal{Q})$ is compact and (6.10) shows that $\left.\mathbf{u}_{m}\right|_{\mathcal{Q}}$ belong to a bounded set of $\mathcal{H}^{\gamma}\left(\mathbf{R} ; V_{g}(\mathcal{Q}), H_{g}(\mathcal{Q})\right)$. Then, proposition 5.2 implies that

$$
\left.\left.\mathbf{u}_{m^{\prime}}\right|_{\mathcal{Q}} \rightarrow \mathbf{u}\right|_{\mathcal{Q}} \text { in } L^{2}\left(0, T ; H_{g}(\mathcal{Q})\right) \text {, strongly } \forall \mathcal{Q} \text {. }
$$


Similarly, for any support $\mathcal{Q}_{j}$ of $\mathbf{w}_{j}$, we have

$$
\left.\left.\mathbf{u}_{m^{\prime}}\right|_{\mathcal{Q}_{j}} \rightarrow \mathbf{u}\right|_{\mathcal{Q}_{j}} \text { in } L^{2}\left(0, T ; H_{g}\left(\mathcal{Q}_{j}\right)\right) \text {, strongly. }
$$

Let $\psi$ be a continuously differentiable function on $[0, T]$ with $\psi(T)=0$. We multiply (6.3) by $\psi(t)$, and then integrate by parts. This leads to the equation

$$
\begin{aligned}
& -\int_{0}^{T}\left(\mathbf{u}_{m}(t), \psi^{\prime}(t) \mathbf{w}_{j}\right) d t+\nu \int_{0}^{T}\left(\left(\mathbf{u}_{m}(t), \mathbf{w}_{j} \psi(t)\right)\right) d t \\
& +\int_{0}^{T} b\left(\mathbf{u}_{m}(t), \mathbf{u}_{m}(t), \mathbf{w}_{j} \psi(t)\right) d t+\int_{0}^{T} b\left(\frac{\nabla g}{g}, \mathbf{u}_{m}(t), \mathbf{w}_{j} \psi(t)\right) d t \\
& =\left(\mathbf{u}_{0 m}, \mathbf{w}_{j}\right) \psi(0)+\int_{0}^{T}\left\langle\mathbf{f}(t), \mathbf{w}_{j} \psi(t)\right\rangle_{g} d t .
\end{aligned}
$$

One should note that each term is same value when we replace $\mathbf{u}_{m}$ by $\left.\mathbf{u}_{m}\right|_{\mathcal{Q}_{j}}$. Therefore, by passing to the limit with the sequence $m^{\prime}$, one obtains from (6.14), (6.15) and (6.16) that

$$
\begin{aligned}
& -\int_{0}^{T}\left(\mathbf{u}(t), \mathbf{v} \psi^{\prime}(t)\right) d t+\nu \int_{0}^{T}((\mathbf{u}(t), \mathbf{v} \psi(t))) d t \\
& +\int_{0}^{T} b(\mathbf{u}(t), \mathbf{u}(t), \mathbf{v} \psi(t)) d t+\int_{0}^{T} b\left(\frac{\nabla g}{g}, \mathbf{u}(t), \mathbf{v} \psi(t)\right) d t \\
& =\left(\mathbf{u}_{0}, \mathbf{v}\right) \psi(0)+\int_{0}^{T}\langle\mathbf{f}(t), \mathbf{v} \psi(t)\rangle_{g} d t
\end{aligned}
$$

Also, we note that the limit holds for $\mathbf{v}=\mathbf{w}_{1}, \mathbf{w}_{2}, \cdots$; by linearity this equation holds for $\mathbf{v}=$ any finite linear combination of the $\mathbf{w}_{j}$, and by a continuity argument (6.17) is still true for any $\mathbf{v} \in V_{g}$. Now writing, in particular, (6.17) with $\psi=$ $\phi \in \mathcal{D}((0, T))$, we see that $\mathbf{u}$ satisfies (6.1) in the distribution sense.

Finally, it remains to prove that $\mathbf{u}$ satisfies (6.2). For this we multiply (6.1) by $\psi$, and integrate. After integrating the first term by parts, we get

$$
\begin{aligned}
& -\int_{0}^{T}\left(\mathbf{u}(t), \mathbf{v} \psi^{\prime}(t)\right) d t+\nu \int_{0}^{T}((\mathbf{u}(t), \mathbf{v} \psi(t))) d t \\
& +\int_{0}^{T} b(\mathbf{u}(t), \mathbf{u}(t), \mathbf{v} \psi(t)) d t+\int_{0}^{T} b\left(\frac{\nabla g}{g}, \mathbf{u}(t), \mathbf{v} \psi(t)\right) d t \\
& =(\mathbf{u}(0), \mathbf{v}) \psi(0)+\int_{0}^{T}\langle\mathbf{f}(t), \mathbf{v} \psi(t)\rangle_{g} d t
\end{aligned}
$$

By comparison with (6.17),

$$
\left(\mathbf{u}(0)-\mathbf{u}_{0}, \mathbf{v}\right) \psi(0)=0 .
$$

We can choose $\psi$ with $\psi(0)=1$; thus

$$
\left(\mathbf{u}(0)-\mathbf{u}_{0}, \mathbf{v}\right)=0, \quad \forall \mathbf{v} \in V_{g},
$$


which implies (6.2). The proof of continuity comes from usual continuity lemma.

7. Uniqueness of SOlutions of Problems 2

Lemma 7.1. If $n=2$, then we have

$$
|b(\mathbf{u}, \mathbf{v}, \mathbf{w})| \cdot c|\mathbf{u}|^{\frac{1}{2}}\|\mathbf{u}\|^{\frac{1}{2}}\|\mathbf{v}\||\mathbf{w}|^{\frac{1}{2}}\|\mathbf{w}\|^{\frac{1}{2}},
$$

for all $\mathbf{u}, \mathbf{v}, \mathbf{w} \in H^{1}\left(\mathbf{R}^{n}\right)$. Also, if $\mathbf{u}$ belong to $L^{2}(0, T ; V) \cap L^{\infty}\left(0, T ; V_{g}\right)$, then $B \mathbf{u}$ belong to $L^{2}\left(0, T ; V_{g}^{\prime}\right)$ and

$$
\|B \mathbf{u}\|_{L^{2}\left(0, T ; V^{\prime}\right)} \cdot 2^{\frac{1}{2}}|\mathbf{u}|_{L^{\infty}(0, T ; H)}\|\mathbf{u}\|_{L^{2}(0, T ; V)} .
$$

If $n=3$, we have

$$
\begin{gathered}
|b(\mathbf{u}, \mathbf{u}, \mathbf{v})| \cdot c\|\mathbf{u}\|_{L^{4}\left(\mathbf{R}^{3}\right)}\|\mathbf{u}\|\|\mathbf{v}\|_{L^{4}\left(\mathbf{R}^{3}\right)} \\
|b(\mathbf{u}, \mathbf{u}, \mathbf{v})| \cdot c|\mathbf{u}|^{\frac{1}{4}}\|\mathbf{u}\|^{\frac{7}{4}}\|\mathbf{v}\|_{L^{4}\left(\mathbf{R}^{3}\right)^{\prime}}
\end{gathered}
$$

Proof. (7.1) and (7.3) come from (2.1) and (4.1), respectively. And (7.2) is from (7.1).

Teorem 7.2. If $n=2$ then the solution of problem 2 given by theorem 1 is unique.

Proof. Let us assume that $\mathbf{u}_{1}$ and $\mathbf{u}_{2}$ are two solutions of problem 2, and let $\mathbf{u}=\mathbf{u}_{1}-\mathbf{u}_{2}$. Then, we have

$$
\begin{aligned}
& \mathbf{u}^{\prime}+\nu A \mathbf{u}+C \mathbf{u}=-B \mathbf{u}_{1}+B \mathbf{u}_{2} \\
& \mathbf{u}(0)=0
\end{aligned}
$$

We take a.e. in $t$ the scalar product of (7.1) with $\mathbf{u}(t)$ in the duality between $V_{g}$ and $V_{g}^{\prime}$. Then one obtains

$$
\begin{aligned}
\frac{d}{d t}|\mathbf{u}(t)|^{2} & +2 \nu\|\mathbf{u}(t)\|^{2}+2 b\left(\frac{\nabla g}{g}, \mathbf{u}(t), \mathbf{u}(t)\right) \\
& =2 b\left(\mathbf{u}_{2}(t), \mathbf{u}_{2}(t), \mathbf{u}(t)\right)-2 b\left(\mathbf{u}_{1}(t), \mathbf{u}_{1}(t), \mathbf{u}(t)\right) \\
& \left.=-2 b\left(\mathbf{u}(t), \mathbf{u}_{2}(t), \mathbf{u}\right)\right)
\end{aligned}
$$

Also, by (7.1), we have

$$
\begin{aligned}
\left.\mid 2 b\left(\mathbf{u}(t), \mathbf{u}_{2}(t), \mathbf{u}\right)\right) \mid & \cdot c|\mathbf{u}(t)|\|\mathbf{u}(t)\|\left\|\mathbf{u}_{2}(t)\right\| \\
& \cdot \nu\|\mathbf{u}(t)\|^{2}+\frac{c^{2}}{\nu}|\mathbf{u}(t)|^{2}\left\|\mathbf{u}_{2}(t)\right\|^{2},
\end{aligned}
$$


and

$$
\begin{aligned}
\left|2 b\left(\frac{\nabla g}{g}, \mathbf{u}(t), \mathbf{u}(t)\right)\right| & \cdot 2 c\|\nabla g\|_{\infty}\|\mathbf{u}\||\mathbf{u}| \\
\cdot & \nu\|\mathbf{u}(t)\|^{2}+\frac{c^{2}}{\nu}\|\nabla g\|_{\infty}^{2}|\mathbf{u}(t)|^{2} .
\end{aligned}
$$

Therefore, we have

$$
\frac{d}{d t}|\mathbf{u}(t)|^{2} \cdot\left(\frac{c^{2}}{\nu}\left\|\mathbf{u}_{2}(t)\right\|^{2}+\frac{c^{2}}{\nu}\|\nabla g\|_{\infty}^{2}\right)|\mathbf{u}(t)|^{2} .
$$

So, one has

$$
\left.\frac{d}{d t} \operatorname{Exp}\left(\int_{0}^{t}\left(\frac{8}{\nu}\left\|\mathbf{u}_{2}(t)\right\|^{2}+\frac{c^{2}}{\nu}\|\nabla g\|_{\infty}^{2}\right) d s\right) \cdot|\mathbf{u}(t)|^{2}\right] \cdot 0 .
$$

Hence, we get

$$
|\mathbf{u}(t)|^{2} \cdot \quad 0, \quad \forall t \in[0, T] .
$$

Thus, $\mathbf{u}_{1}=\mathbf{u}_{2}$.

For the case $n=3$, we have different theory. that

Theorem 7.3. If $n=3$, then there is at most one solution of problem 2 such

$$
\begin{gathered}
\mathbf{u} \in L^{2}\left(0, T ; V_{g}\right) \cap L^{\infty}\left(0, T ; H_{g}\right), \\
\mathbf{u} \in L^{8}\left(0, T ; L^{4}\left(\mathbf{R}^{3}\right)\right) .
\end{gathered}
$$

Proof. Let us assume that $\mathbf{u}_{1}$ and $\mathbf{u}_{2}$ are two solutions of problem 2 which satisfies (7.7) and (7.8) and let $\mathbf{u}=\mathbf{u}_{1}-\mathbf{u}_{2}$. Then, by (7.5)

$$
\begin{aligned}
& \frac{d}{d t}|\mathbf{u}(t)|^{2}+2 \nu\|\mathbf{u}(t)\|^{2}+2 b\left(\frac{\nabla g}{g}, \mathbf{u}(t), \mathbf{u}(t)\right) \\
= & \left.-2 b\left(\mathbf{u}(t), \mathbf{u}_{2}(t), \mathbf{u}\right)\right)=2 b\left(\mathbf{u}(t), \mathbf{u}(t), \mathbf{u}_{2}(t)\right) .
\end{aligned}
$$

Now, we have from (7.4) and young inequality $\left(p=\frac{8}{7}, q=8, \epsilon=\nu\right)$ that

$$
\left|2 b\left(\mathbf{u}(t), \mathbf{u}(t), \mathbf{u}_{2}(t)\right)\right| \cdot \nu\|\mathbf{u}(t)\|^{2}+\frac{c^{8}}{\nu^{7}}|\mathbf{u}(t)|^{2}\left\|\mathbf{u}_{2}(t)\right\|_{L^{4}\left(\mathbf{R}^{3}\right)}^{8} .
$$

So, by (7.6), (7.9) and (7.10), one obtains that

$$
\frac{d}{d t}|\mathbf{u}(t)|^{2} \cdot\left(\frac{c^{8}}{\nu^{7}}\left\|\mathbf{u}_{2}(t)\right\|_{L^{4}\left(\mathbf{R}^{3}\right)}^{8}+\frac{c^{2}}{\nu}\|\nabla g\|_{\infty}^{2}\right)|\mathbf{u}(t)|^{2} .
$$


So, one has

$$
\left.\frac{d}{d t} \operatorname{Exp}\left(\int_{0}^{t}\left(\frac{c^{8}}{\nu^{7}}|\mathbf{u}(t)|^{2}\left\|\mathbf{u}_{2}(t)\right\|_{L^{4}\left(\mathbf{R}^{3}\right)}^{8}+\frac{c^{2}}{\nu}\|\nabla g\|_{\infty}^{2}\right) d s\right) \cdot|\mathbf{u}(t)|^{2}\right] \cdot 0 .
$$

Hence, we get

$$
|\mathbf{u}(t)|^{2} \cdot 0, \quad \forall t \in[0, T] .
$$

Thus, $\mathbf{u}_{1}=\mathbf{u}_{2}$.

\title{
REFERENCES
}

1. P. Constantin and C. Foias, Navier-Stokes equations, Chicago Lectures in Mathematics, The University of Chiago Press, 1988.

2. P.-L. Lions, Mathematical topics in fluid mechanics, Volume 1 Incompressible modesl, Clarendon Press, 1996.

3. J. Roh, $g$-Navier-Stokes equations, Thesis, University of Minnesota, 2001.

4. G. R. Sell and Y. You, Dynamics of evolutionary equations. Applied Mathematical Sciences, 143. Springer-Verlag, New York, 2002.

5. R. Temam, Navier-Stokes equations: theory and numerical analysis, 1977.

\author{
Hyeong-Ohk Bae \\ Department of Mathematics, \\ Ajou University, \\ Suwon 442-479, \\ Republic of Korea \\ E-mail: hobae@ajou.ac.kr \\ Jaiok Roh \\ Department of Mathematics, \\ Hallym University, \\ Chuncheon, \\ Republic of Korea \\ E-mail: joroh@dreamwiz.com
}

